\title{
GENERATION OF THETA RHYTHM IN MEDIAL ENTORHINAL CORTEX OF FREELY MOVING RATS
}

\author{
SUSAN J. MITCHELL* and JAMES B. RANCK, JR.
}

Department of Physiology, Downstate Medical Center, State University of New York, Brooklyn, N. Y. 11203 and Department of Physiology, University of Michigan, Ann Arbor, Mich. 48109 (U.S.A.)

(Accepted October 4th, 1979)

Key words: theta rhythm - entorhinal cortex - hippocampus - dentate gyrus - voluntary behaviors - paradoxical sleep

\section{SUMMARY}

A regular slow wave theta rhythm can be recorded in the medial entorhinal cortex (MEC) of freely moving rats during voluntary behaviors and paradoxical sleep. Electrode penetrations normal to the cortical layers proceeding from the deeper to the more superficial layers reveal a continuous theta rhythm in layers IV-III (deep MEC theta rhythm) with an amplitude maximum in layer III, a null between the outer onethird of layer III and the inner one-half of layer I, and a continuous phase-reversed theta rhythm in layers II-I (superficial MEC theta rhythm) with an amplitude maximum there. Deep MEC theta rhythm is similar in phase and wave shape to CA1 theta rhythm; superficial MEC theta rhythm is similar in phase to DG theta rhythm. Laminar profiles throughout MEC show that the theta rhythm is generated there; it is not volume conducted from hippocampus.

\section{INTRODUCTION}

A regular slow wave theta rhythm can be recorded from the hippocampus of freely moving rats $5,45,49$, rabbits $^{51}$ and cats ${ }^{2,7,47}$ during some waking behaviors and during paradoxical sleep. The theta rhythm can also be recorded in the anesthetized or curarized animal following appropriate stimuli $4,5,13,33,50$. Early investigations suggested that the hippocampal generator, i.e. the source and sink of current flow which gives rise to the extracellular potential, was located in pyramidal cells of CA114,15. More recent work has shown that there are two generators of theta rhythm in the freely moving rat ${ }^{5,49}$ and rabbit ${ }^{51}$ and in anesthetized or curarized rat ${ }^{5,50}$ and rabbit $^{4}$. The generators are located in the CAl and dentate gyrus regions of hippocampus.

* Present address for all correspondence: Department of Psychology, Johns Hopkins University, Baltimore, Md. 21218, U.S.A. 
A theta rhythm can also be recorded in entorhinal cortex of cats 2,20 . The present study was undertaken to describe the slow wave activity in the entorhinal cortex of freely moving rats. The entorhinal cortex is the source of a large and topographically organized fiber projection to the hippocampus and it is the recipient of an orderly multisynaptic projection from the Ammon's horn fields of hippocampus. We report here that a theta rhythm can be recorded in medial entorhinal cortex (MEC) and we show that there is at least one theta rhythm generator in MEC. Some aspects of entorhinal cortex anatomy which are important to this study are reviewed.

\section{ANATOMY}

Entorhinal cortex is located between isocortex and hippocampus (Figs. 1 and 2). It has a distinct dorso-medial area (MEC) and a ventrolateral area (lateral entorhinal cortex or LEC) $3,21,24$. Histochemical stains of entorhinal cortex confirm this division into medial and lateral areas and extend the number of divisions to include transition zones between MEC and $\operatorname{LEC}^{9,10,17,38,43}$. Fig. 1 illustrates the subdivisions of entorhinal cortex as determined by both cytoarchitectonics and the Timm sulfide silver stain for heavy metals.

The nomenclature of Ramón y Cajal ${ }^{6}$ is used here to describe the cortical layers (Fig. 2C). Axons from star cells in layer II, pyramidal cells in layers III, V, VI, and VII and globular and polygonal cells of layer VII constitute the efferent projection of entorhinal cortex ${ }^{24}$. It is important to note that the dendrites of layer II star cells remain for the most part within the second layer with few ascending to the first layer ${ }^{24}$. Pyramidal cells located in layer III have some dendritic processes in that layer, none in layer II and extensive dendritic branching in layer I. Lorente de Nó ${ }^{24}$ has estimated that 'the proportion of the number of synapses of the pyramid cells in the third and first layer to those in the second layer must be some 1000 to 1.' In addition to the

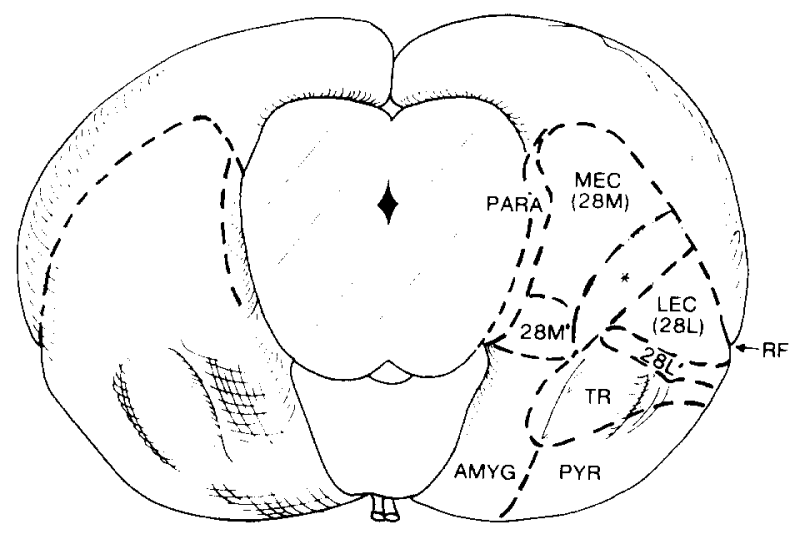

Fig. 1. Caudo-ventral view of the rat brain sectioned in the coronal plane at the level of the colliculi. Cortical subdivisions are indicated by dashed lines on the right side of the figure. Abbreviations are: for medial entorhinal cortex (MEC or 28M), lateral entorhinal cortex (LEC or 28L) transition area between MEC and LEC $\left(^{*}\right)$, parasubiculum (PARA), ventral subdivisions of EC according to Haug ${ }^{17}$ (28M' $\left.\mathrm{M}^{\prime}, 28 \mathrm{~L}^{\prime}\right)$, transition area between EC, pyriform cortex, and amygdala (TR), pyriform cortex (PYR), amygdala (AMYG), and rhinal fissure (RF). Redrawn from Blackstad ${ }^{3}$ and Haug $^{17}$. 

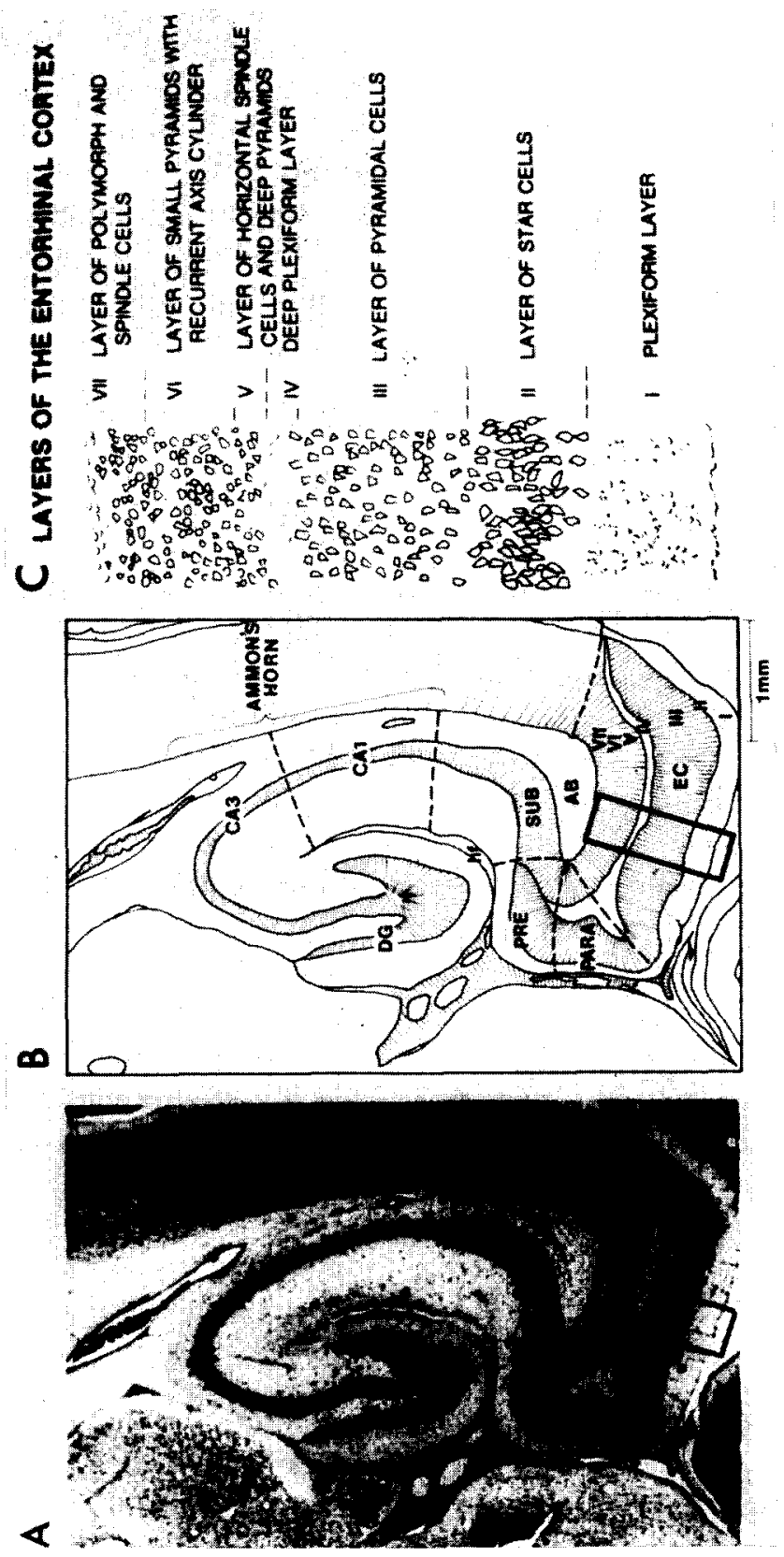

Fig. 2. A: photograph of a horizontal section through the hippocampal formation of the rat brain at a level half way between dorsal and ventral limits of MEC (Fig. 1). B: trace of the photograph in A with areas labeled. The hippocampal formation includes dentate gyrus (DG), Ammon's horn (CA1 and CA3), subiculum (SUB), presubiculum (PRE), parasubiculum (PARA), and entorhinal cortex (EC). Other abbreviations are: for angular bundle (AB) and hippocampal fissure (hf). C: trace of the cells of EC from the area marked in A and B. Bottom of the trace is the pial surface, top is angular bundle. The layers are named for the most common cell type found in accordance with Ramón y Cajal's scheme ${ }^{6}$. He named layer $\mathrm{V}$ for the horizontal cells which he was able to stain; Lorente de Nó ${ }^{24}$ using Golgi and Cox stains described the layer in much greater detail and found that pyramidal cells were the most common cell type. Therefore this layer was named for both horizontal and pyramidal cells. The relative thickness of the cell layers varies along dorso-ventral and medio-lateral axes of EC. 
predominant cell type, interneurons which have axons either ascending, ramifying locally, or descending are found in all layers.

The largest efferent projection of EC is to Ammon's horn and the dentate gyrus. This projection has two divisions. The first is a predominantly ipsilateral projection to DG granule cells and the distal apical dendrites of CA3 pyramidal cells $8,18,19,31,40,46$. The projection to DG granule cells originates from EC layer II star cells; the cells of origin of the EC projection to CA3 pyramidal cells are not known ${ }^{41}$. The second division is a bilateral projection (equally dense on both sides) to the apical dendrites of CA1 pyramidal cells ${ }^{40,41,46}$. This projection originates from EC layer III pyramidal cells $^{41,42}$.

\section{METHODS}

Sixteen male Long Evans hooded rats which weighed 350-450 $\mathrm{g}$ at the beginning of the training period were used for the study. The animals were trained over a period of 8 days to walk in an enclosed treadmill. Our observations and the observations of others $^{25}$ indicate that both frequency and amplitude of theta rhythm increase with increasing speed of locomotion. We thus attempted to maintain both amplitude and frequency of theta rhythm constant during each episode of locomotion by having the rat walk at a constant speed of $20 \mathrm{~cm} / \mathrm{sec}$ at an even pace, never pausing or rearing up on his hind legs.

After the animals had been trained, electrodes were surgically implanted. The rats were anaesthetized with sodium pentobarbital at $40 \mathrm{mg} / \mathrm{kg}$ body weight. Three small holes were drilled, one through each of the frontal bones and one through the interparietal bone over cerebellum. Stainless steel self-tapping screws (TX 2-4 Small Parts, Inc., Miami, Fla., cut off to a length of $\frac{3}{8}$ in.) were screwed firmly into the drilled holes. Silver wires were wound around the shaft of the screw between the bone and the head of the screw and led to a Cannon connector (MIKO-1-7SH, ITT Cannon Elec). An additional silver wire was inserted into the neck muscles and led to the connector; this wire served to ground the rat. The frontal connections were used to record neocortical EEG differentially and one of them also served as the indifferent electrode for recording signals from hippocampus and entorhinal cortex. The interparietal connection was available for use as an indifferent electrode in case either of the frontal connections failed.

Each rat had either one electrode (aimed at dentate gyrus) or two electrodes (one aimed at dentate gyrus, the other at CA1) for recording hippocampal slow waves. With the rat's skull level in the stereotaxic device (bregma and lambda in the same horizontal plane) the hippocampal electrodes were placed $4.2 \mathrm{~mm}$ posterior to bregma and $2.5-2.8 \mathrm{~mm}$ lateral to the midline. A fixed electrode lowered $2.5-3.25 \mathrm{~mm}$ from the surface of the skull was used to record slow waves from CAl and either a fixed electrode lowered $3.5 \mathrm{~mm}$ from surface of skull or a movable electrode was used to record slow waves from dentate gyrus.

The movable electrode used for dentate gyrus records consisted of two parts: a threaded nylon rod (length $1.6 \mathrm{~cm}$, TRN 032, Small Parts Inc., Miami, Fla.) tapped to 

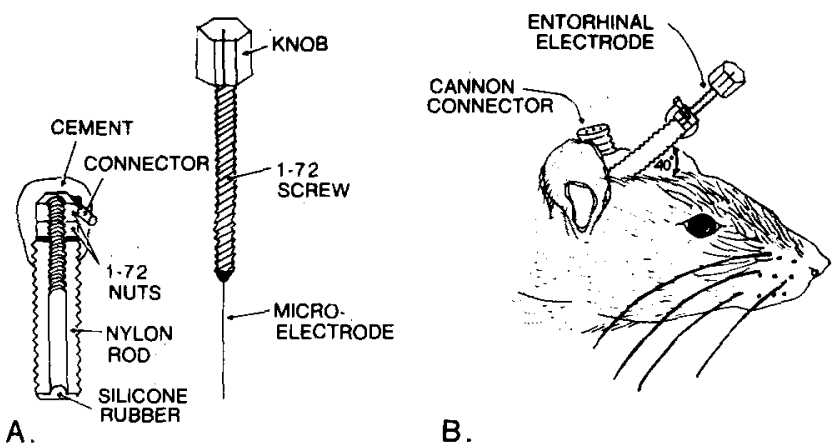

Fig. 3. A: the two parts of the movable microelectrode. The nylon part is illustrated in cross section. B: orientation of the movable entorhinal electrode on the rat.

receive a 1-72 stainless steel screw and extended at the top with two 1-72 stainless steel hex nuts to which a female Cannon socket connector had been soldered (the nuts were cemented in place), and a 1-72 stainless steel screw with a knob on one end and with a hole $1 \mathrm{~mm}$ deep in the other end into which the actual electrode was soldered (Fig. 3A). A thin layer of silicone rubber (3110 RTV, Dow Corning, Midland, Mich.) was applied to the bottom of the drilled out nylon rod to prevent fluid from seeping up into it. During the surgery, the drilled out nylon rod was placed stereotaxically over a small hole in the skull and cemented into place. The screw carrying the electrode was inserted into the nylon rod just before the first recording sessions then left in place for the duration of the study.

Each rat also had a second movable electrode aimed for entorhinal cortex on the side contralateral to the hippocampal electrodes. This electrode was aimed at a point $5.2 \mathrm{~mm}$ lateral, $5.0 \mathrm{~mm}$ dorsal, and $0.8 \mathrm{~mm}$ anterior to earbar zero (rat's skull level as previously described). To reach this point the electrode holder was placed $5.2 \mathrm{~mm}$ lateral and then tilted anteriorly in the sagittal plane so that the angle formed in this plane by the electrode holder and the skull surface was $40^{\circ}$ (Fig. 3B). It was cemented into place. This particular approach was selected for 3 reasons: first, it allowed maximum stability of the recording electrode; second, it allowed the electrode to pass through entorhinal cortex in a direction normal to the cortical layers; third, it allowed advancement of the electrode to the entorhinal cortex without crossing either the hippocampal fissure, which is rich with blood vessels, or the transverse sinus.

The movable electrode already described was used for the entorhinal penetrations on the first few animals but because the electrode rotated as it was lowered into brain it was found to cause extensive tissue damage along this particularly long and deep track, especially if it was not exactly centered in its screw. Therefore, a second type of movable electrode which did not rotate was used for subsequent entorhinal penetrations. It is a version of the Harper and McGinty ${ }^{16}$ electrode modified to carry a single large electrode, and it was placed exactly as already described.

The fixed electrodes were prepared from insulated blunt cut $150 \mu \mathrm{m}$ Nichrome wire (Driver-Harris Co., Harrison, N.J.). Movable electrodes were prepared from 225 $\mu \mathrm{m}$ Superturalloy spring stainless steel wire (Turotech, Stamford, Conn.) etched over 1 
$\mathrm{mm}$ to a tip diameter of approximately $5 \mu \mathrm{m}$ coated with Epoxylite (Epoxylite Corp., Anaheim, Calif.) and each coat baked at $135^{\circ} \mathrm{C}$ for $5 \mathrm{~h}$ until 8 coats had been applied These electrodes were then ground to a blunt tip of diameter $80-125 \mu \mathrm{m}$, using a grinding wheel on a Dremel tool. These electrodes recorded multiunit activity in addition to slow waves. They were usually too large to record single unit activity.

After the surgery, antibiotic ointment was applied to the wound, and $0.1 \mathrm{ml}$ of Bicillin (Wyeth, 300,000 units/ml) administered i.m. to the rat. A second dose of Bicillin was administered 3 days later. The animals were allowed to recover from surgery for 7 days before data collection began.

Signals from the electrodes were led to a headstage which was firmly attached to the Cannon connector of the rat's implant. Field effect transistors in a source follower configuration in the headstage were used to eliminate movement artifact. The signals were then led to conventional recording devices. The slow waves from the neocortical, dentate gyrus, CA1, and entorhinal electrodes were filtered at 0.3 or $1-75 \mathrm{~Hz}$ and recorded by a Grass polygraph. The slow waves from dentate gyrus, CA1, and the entorhinal electrode all filtered at 0.3 or $1-500 \mathrm{~Hz}$, were simultaneously recorded on magnetic tape. The entorhinal signal was also passed through a second order active filter $(500-40 \mathrm{kHz},-12 \mathrm{~dB} /$ octave) so that action potentials of the cells could be monitored on an oscilloscope and loudspeaker and recorded on magnetic tape.

Video tapes of two rats were made in which part of the screen showed the rats" behavior, and part showed simultaneously occurring slow waves from dentate gyrus, CAl, and entorhinal cortex as they were written on polygraph paper. The behaviors which were taped included walking in the treadmill, walking around an open field, turning, rearing up on hind legs, freezing, lying down motionless, grooming, scratching, eating, drinking, climbing into and out of the home cage, and being handled while struggling and while relaxing.

The average frequency of entorhinal, dentate gyrus, and CAl theta rhythms was calculated after measuring the duration (D) on the polygraph paper of at least 50 cycles $(\mathrm{N})$ of theta at each increment along the entorhinal penetration and then dividing $\mathrm{N}$ by $\mathrm{D}$.

Data collected from the rat at each increment along the entorhinal penetration were used to construct amplitude and phase profiles of the theta rhythm recorded within the layers of entorhinal cortex.

(a) Amplitude. Amplitude of entorhinal theta was calculated by measuring the peak to peak values of at least 50 individual cycles of theta recorded on the polygraph paper at each increment of the penetration then averaging them. Standard deviations (S.D.) and standard errors of the mean (S.E.M.) were also calculated.

Because the walking behavior of the animal was controlled and all data were recorded during episodes of locomotion at $20 \mathrm{~cm} / \mathrm{sec}$ the amplitude of the reference signal (DG theta rhythm) was found to remain reasonably constant (see Results). It was therefore not necessary to normalize the entorhinal theta rhythm amplitude to control for overall changes in the theta rhythm from one episode of locomotion to the next.

(b) Phase. The construction of the phase profile depends on the determination of 
the phase relation between the entorhinal electrode and the fixed reference electrode. Theta rhythm has been shown to be generated in both CA1 and dentate gyrus in the rat $5,49,50$. Dentate gyrus was chosen as the site for the reference electrode because the theta rhythm recorded there is greater in amplitude ${ }^{4,49}$ and more stable than that recorded from $\mathrm{CA} 1^{4,5}$. The phase relation between entorhinal and dentate gyrus theta rhythms was determined by a cross-correlation technique ${ }^{50}$. The data recorded on magnetic tape were amplified and filtered to $20 \mathrm{~Hz}$ ( $-24 \mathrm{~dB} /$ octave) using a two channel low pass maximum flat active filter (Khron-Hite model 3322R).

To measure phase relations and wave shape relations, cross-correlation computations were used. The entorhinal and dentate gyrus signals filtered to $20 \mathrm{~Hz}$ were digitized at $1 \mathrm{msec}$ intervals using a PDP 11/45 computer. If the entorhinal signal was approximately phase-reversed with respect to the dentate gyrus signal, the dentate signal was electronically inverted before cross-correlation computations were calculated. The digitized signals were displayed on a graphics terminal in continuous segments of $300 \mathrm{msec}$ each. A decision was made as to whether a cross-correlation computation would be performed. If theta rhythm was absent in either signal the data were rejected. If the data were accepted, a series of cross-correlation coefficients were calculated as described in detail by Winson ${ }^{50}$. Briefly, the maximum correlation coefficient and the number and direction of msec intervals necessary to displace the signals from the real time position to the position which yielded the maximum correlation coefficient was stored for each segment analyzed. At least 50 segments were analyzed at each increment along the entorhinal penetration. The computer printed out the maximum correlation coefficients and leads or lags as well as averages, standard deviations and standard errors of the mean of these values.

At the end of the experiment the location of the reference electrodes and a selected location along the entorhinal track were marked by passing $2-3 \mu \mathrm{A}$ of current for $5 \mathrm{sec}$, and developing the mark by the Prussian blue technique. The animals were deeply anesthetized then perfused transcardially first with saline then with $10 \%$ formalin in saline containing $2 \%$ potassium ferrocyanide. After sufficient fixation in $10 \%$ formalin in saline the brains were put into $30 \%$ sucrose and refrigerated for several days until the tissue sank. Each brain was cut in half in the coronal plane just posterior to the place where the reference electrodes had entered the tissue. Frozen sections of $40 \mu \mathrm{m}$ each were cut in the horizontal plane for the posterior portion of the brain and in the coronal plane for the anterior portion. The sections were stained with cresyl violet.

To localize the electrophysiological data to discrete layers of MEC both the length of the track in the animal and the number of histological sections in which the track appeared in MEC were determined. The length of the track in the animal was determined by knowing at which increment the electrode entered MEC and at which increment the track was terminated. The microelectrode either passed from isocortex directly into MEC or it passed from neocortex through the fiber bundle into MEC depending on the medio-lateral coordinate of the penetration. In the first case MEC was identified by the change in the firing pattern of the cells between isocortex and $\mathrm{MEC}$; in the second case, since no action potentials were recorded as the micro- 
electrode passed through the fiber bundle, when action potentials were recorded the electrode had entered the deepest layers of MEC. The electrophysiological data were then fit to the histological data by equating the length of the track in the fixed tissue and the length of the track in the animal. Each track contained one Prussian blue mark at a key point which helped to assure a proper fit of the data. This technique has an error of approximately $90 \mu \mathrm{m}$ or $2-3$ fixed sections.

\section{RESULTS}

The results of this study show that theta rhythm can be recorded from MEC of the rat. In the deeper cortical layers it is approximately phase-reversed to dentate gyrus theta rhythm (approximately in phase with CA1 theta rhythm) and in the more superficial layers it undergoes a rapid phase reversal so that it becomes approximately in phase with dentate gyrus theta rhythm.

\section{Behavioral correlate}

Both direct observation of the rats and observation of the video tapes made of the rats gave no indication that the behavioral correlate of entorhinal theta rhythm (both deep and superficial) differed from the behavioral correlate of CAl and dentate gyrus theta rhythms which were recorded simultaneously. During waking behaviors entorhinal theta rhythm was observed to be present during walking, turning, rearing up on hind legs, climbing into and out of the home cage and struggling. It was absent during freezing, eating, drinking, and grooming. It was present during paradoxical sleep and absent during slow wave sleep. Thus it was present during movements which may be described as 'voluntary movements' 45 and during paradoxical sleep.

A.

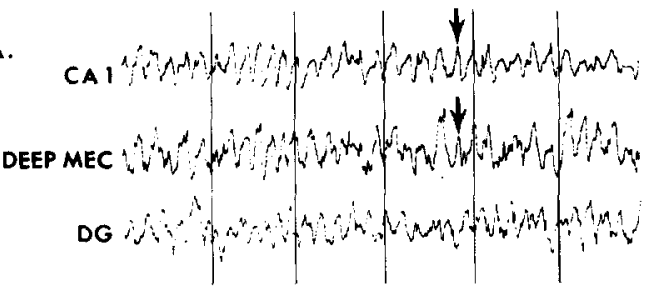

B.

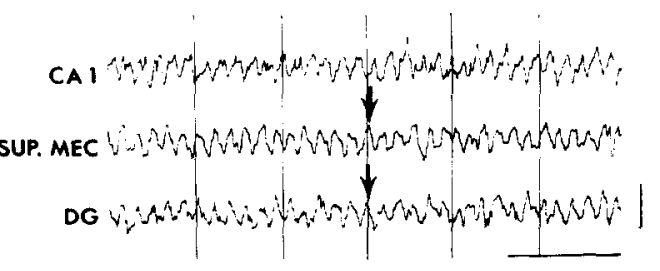

Fig. 4. A: theta rhythm from deep MEC as compared to simultaneously recorded CA1 and DG theta rhythms. Deep MEC theta rhythm is approximately in phase with CA1 theta rhythm. Arrows indicate a place where for a few cycles deep MEC theta rhythm is more similar to CA1 than to DG theta rhythm. B: theta rhythm from superficial MEC as compared to simultaneously recorded CA1 and DG theta rhythms. Superficial MEC theta rhythm is approximately in phase with DG theta rhythm. Arrows indicate a place where for a few cycles deep MEC theta rhythm is more similar to DG than to CA1 theta rhythm. Calibration bars $1 \mathrm{sec}$ and $500 \mu \mathrm{V}$. 


\section{Frequency}

The theta rhythm is a sinusoidal signal with considerable frequency modulation. To determine the phase relation between two different signals it must be shown that the two signals have similar frequency modulation. It was obvious by inspection of the polygraph records that the theta rhythms recorded in both CA1 and DG, and deep and superficial MEC, had similar frequency modulation (Fig. 4). The average frequency of entorhinal theta rhythm over all animals was $7.08 \mathrm{~Hz}$, S.D. 0.29 (range 6.48-7.41 Hz, S.D. 0.23-0.34). In 3 animals the frequency of the dentate gyrus theta rhythm was calculated and in two different animals the frequency of CA1 theta rhythm was calculated at the same time over which the entorhinal calculations were made. The average frequency of hippocampal (DG and CA1) theta rhythm over all animals was $7.11 \mathrm{~Hz}$, S.D. 0.32 (range $6.49-7.35 \mathrm{~Hz}$, S.D. 0.25-0.32).

\section{Amplitude}

Although there is cycle by cycle variation in the peak to peak amplitude of the dentate gyrus theta rhythm, there is very little variation in the average amplitude recorded during different episodes of locomotion. In one rat the average peak to peak amplitude of 50 cycles of dentate gyrus theta rhythm was compared at 6 evenly spaced intervals during the experiment. The overall average of these values was $978 \mu \mathrm{V}$ (range 920-1068 $\mu \mathrm{V}$, S.D. 174-227). Data were not normalized to control for variation in amplitude between episodes of locomotion because these variations were so small.

Dentate gyrus theta rhythm is approximately phase-reversed to CA1 theta rhythm and amplitude correlations between the two signals have a negative value $\mathrm{e}^{50}$. Deep entorhinal theta rhythm is approximately in phase with CA1 theta rhythm. The waveform of deep MEC theta rhythm is more similar to CA1 theta rhythm than to dentate theta rhythm (see wave shape).

\section{Wave shape}

For two sets of data from two different rats, correlation coefficients were calculated at selected sites between entor hinal theta rhythm and both CA1 and dentate gyrus theta rhythms. These correlation coefficients $(r)$ were higher when the entorhinal signal was compared to the hippocampal signal which was approximately in phase with it. For deep entorhinal vs CA1 the $r$ range was $0.83-0.92$ (6 values), for deep entorhinal vs DG the $r$ range was $0.68-0.78$ (6 values); for superficial entorhinal vs DG the $r$ range was $0.75-0.82$ ( 3 values) and for superficial entorhinal vs CA1 the $r$ range was $0.75-0.77$ ( 3 values). Using the identical program Winson ${ }^{50}$ has shown that the average value for maximum cross-correlation coefficient of CA1 theta rhythm recorded at two sites in the same coronal plane (or DG theta rhythm recorded at two sites in the same coronal plane) is 0.95 . These signals are termed isomorphic. The average values for maximum cross-correlation coefficients for deep entorhinal vs CA1 theta rhythms are not in the range of those values reported for isomorphic signals, but the values are higher than those values found for deep entorhinal vs DG theta rhythms. Likewise, the average values for maximum cross-correlation coefficients for superficial entorhinal vs DG theta rhythm are not in the range reported for isomorphic 
signals and although the values suggest that superficial entorhinal theta rhythm might be more similar to dentate gyrus theta rhythm than to CAl theta rhythm, there is not adequate data to support this claim.

\section{Laminar profiles}

Data from 7 animals were used to construct laminar profiles of MEC theta rhythm. Fig. 5 is a representative profile. No theta rhythm was recorded as the electrode passed through cellular areas of isocortex at the beginning of the penetration. In the more medial penetrations the electrode passed through the fibers of angular bundle before entering the deep layers of MEC. In the angular bundle and in MEC layers VII-V, a low amplitude intermittent theta rhythm approximately in phase with CA1 theta rhythm was recorded. A continuous theta rhythm was recorded within layers IV-III where the average value for the amplitude reached a maximum (Fig. 6). This deep MEC theta rhythm was approximately phase-reversed to DG theta rhythm (approximately in phase with CA1 theta rhythm). Phase relations between MEC, CA1, and DG theta rhythms were determined by direct observation of the polygraph record and in 5 cases the phase relations between the electronically inverted DG theta rhythm and the deep MEC theta rhythm were computed. In 4 cases deep MEC theta rhythm led the inverted DG theta rhythm by average values between 5.3 and $17.3 \mathrm{msec}$ (about $13.25-43.25^{\circ}$ ). In one case deep MEC theta rhythm lagged DG theta rhythm by an average value of $3.5 \mathrm{msec}$ (about $8.75^{\circ}$ ). Once a lead or lag was established it remained almost constant until the point of phase reversal. Although part of measured phase

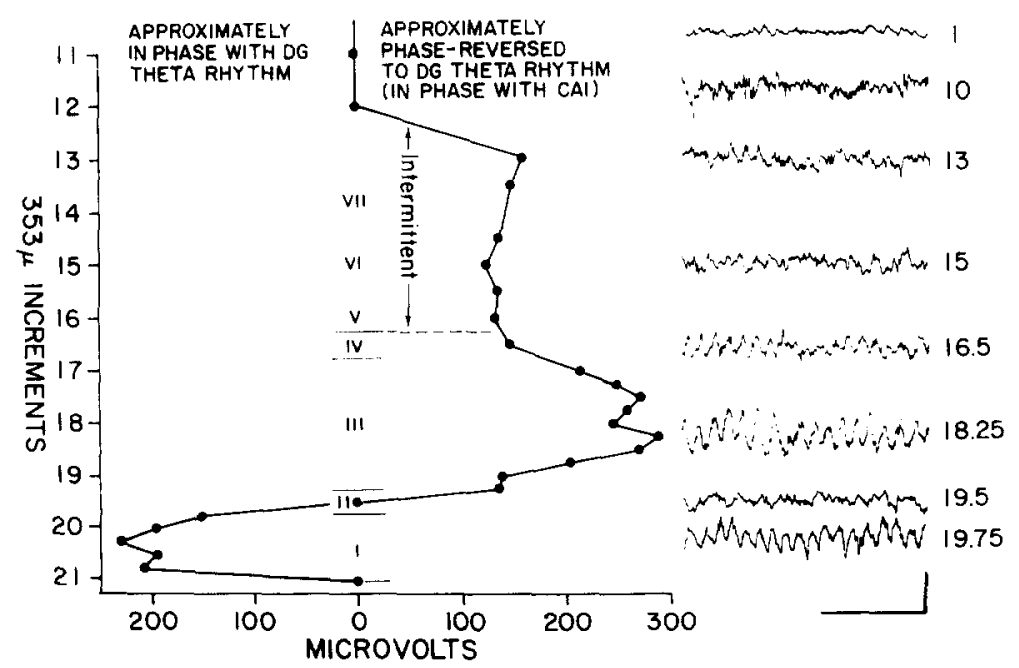

Fig. 5. Laminar profile of the MEC theta rhythm and actual data recorded on the MEC electrode. Theta rhythm phase and amplitude are plotted versus distance along the electrode penetration as measured in $353 \mu \mathrm{m}$ increments. The microelectrode passed through isocortex for the first 13 increments. No theta rhythm can be seen in data recorded at increments 1 and 10 shown in the upper right corner of the figure. The other segments of actual data are shown opposite the increment at which they were recorded. The MEC layers through which the electrode passed at each increment are also shown on the profile. See text for additional details. Calibration bars $1 \mathrm{sec}$ and $500 \mu \mathrm{V}$. Rat H-68. 
A

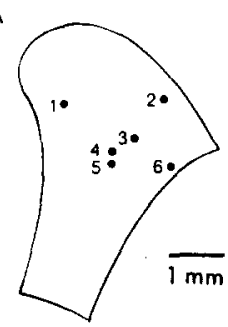

B

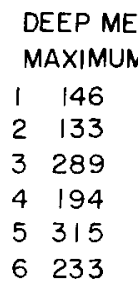

SUP. MEC

MAXIMUM

242

|3 I

231

218
RAT

H -90

$\mathrm{H}-97$

H- 68

$\mathrm{H}-74$

$\mathrm{H}-98$

$H=76$

Fig. 6. A: caudo-ventral view of MEC as in Fig. 1. Dots mark the terminations of six penetrations made through MEC. B: the numbers associated with each penetration are the maximum values in $\mu \mathrm{V}$ for deep and superficial MEC theta rhythm. In rat H-90 the deep peak was recorded at the medial border of MEC. The low values for H-97 are attributed to excessive cell damage around the electrode track. Penetrations in H-74 and H-76 were terminated immediately after the phase reversal. The data from rat H-81 (Deep 212/Sup 167) could not be placed in MEC because an isocortical lesion caused great distortion of the retrohippocampal area. No systematic change in peak amplitudes is seen in either the dorso-ventral or the medio-lateral directions.

difference is due to individual differences in the shapes of the waves being compared, phase comparison data can nevertheless still reveal the existence of a gradual phase shift like the one which exists in CA1 in hippocampus ${ }^{50}$. There is no suggestion that deep MEC theta rhythm undergoes a gradual shift in phase before the sudden reversal.

The amplitude of theta rhythm declined as the electrode approached layer II and a null signal was recorded in a zone located between the outermost portion of layer III and the innermost portion of layer I. As the electrode moved on toward the pial surface a continuous theta rhythm was again recorded but it was approximately in phase with DG theta rhythm. The average value for the amplitude of this superficial MEC theta increased to a maximum in layers II-I (Fig. 6). For two penetrations the phase relation between superficial MEC and DG theta rhythm was computed. In both cases superficial MEC theta rhythm lagged DG by relatively constant average values of 4.0-6.4 msec (about $6.5^{\circ}$ ).

The phase reversal between deep and superficial MEC theta rhythm was complete within $90-180 \mu \mathrm{m}$. Computer analysis of 5 of the penetrations yielded values for the phase reversal of $120-167^{\circ}$. Because of the contribution from differences in wave shape, it is not surprising that the phase relation between MEC and DG theta rhythm varies from animal to animal by as much as $52^{\circ}$ and that the phase reversals are not exactly $180^{\circ}$. Some of this variation might also be due to variation in phase of the reference theta rhythm with electrode placement in DG. Winson ${ }^{50}$ reports that signals recorded in a single rostro-caudal position in the hippocampus were generally either in phase or phase-reversed within $10^{\circ}$, but when electrodes were in different rostro-caudal placements deviations up to $14.3 \mathrm{msec}$ (about $36^{\circ}$ ) were encountered between isomorphic signals.

The best evidence for the location of the phase reversal comes from one study (Fig. 7) in which the electrode traveled at the border of entorhinal cortex and parasubiculum through layer III then entered layer II and traveled through it at the point where this layer is widest. Layer II is $2-3$ times wider at the medial boundary of 


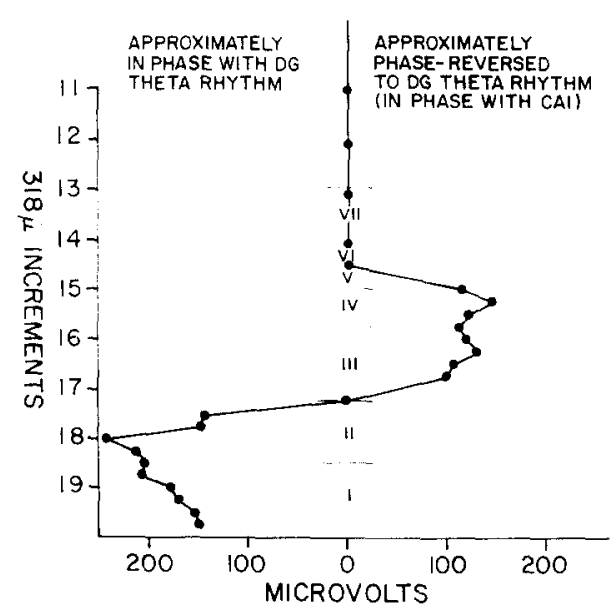

Fig. 7. Laminar profile of data from rat H-90. The electrode traveled through isocortical cellular layers entering MEC at its medial boundary. A low amplitude theta rhythm with maximum amplitude in layers IV-III was recordeo between increments 15 and 17. A null signal was recorded between layers III and II, and a phase-reversed theta rhythm with maximum amplitude in layer II was recorded between increments 17.5 and 19.75 . See text for additional details.

MEC than anywhere else in MEC (Fig. 2). Thus the electrode remained in layer II for a distance 2-3 times longer than that along all the other penetrations. In this animal the histological fit clearly shows that the phase reversal occurred at the border between layers III and II or in the deep half of layer II.

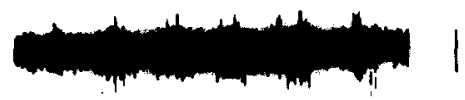

A
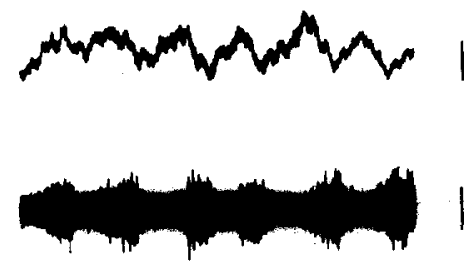

B

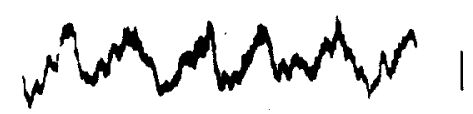

Fig. 8. A: upper trace, a multiunit recording of theta cells in MEC layer III during locomotion; lower trace, slow waves recorded simultaneously from the same electrode. B: upper trace, a multiunit recording of theta cells recorded in MEC layer III during paradoxical sleep; lower trace, slow waves recorded simultaneously from the same electrode. This electrode track was at the medial border of MEC, rat H-90. Calibration bars, upper traces, $125 \mu \mathrm{V}$; lower traces, $200 \mu \mathrm{V}$, and $0.1 \mathrm{sec}$. 
Action potentials from cells were also recorded by the entorhinal electrode at each increment along the penetration. Theta cells, defined as cells which have the same behavioral correlate as the theta rhythm and which increase their rates of firing only when a theta rhythm is present ${ }^{\mathbf{3 0 , 3 5}}$ are found in MEC (Fig. 8). These cells are always found in layer III in the vicinity of the maximum theta rhythm amplitude. Although the data suggest that theta cells are scarce or absent in the deeper layers, the data are inadequate to make this claim. It is also not clear whether theta cells are found in the superficial layers. Others ${ }^{1}$ have observed similar cells.

\section{Paradoxical sleep}

No laminar profiles were constructed by recording theta rhythm during paradoxical sleep at each increment along a penetration. However, in two animals theta rhythm was recorded during paradoxical sleep at one site in the vicinity of the deep entorhinal theta rhythm peak, and in one animal at one site in the vicinity of the superficial entorhinal theta rhythm peak. In all cases the phase relation between the entorhinal and dentate signals was approximately the same during locomotion and paradoxical sleep. Amplitude of entorhinal theta rhythm was $10-60 \%$ greater during paradoxical sleep than during locomotion.

\section{DISCUSSION}

\section{Volume conduction}

If the theta rhythm was volume conducted to MEC it would probably show an attenuation in amplitude in either the medio-lateral or dorso-ventral directions or both, and it would be unlikely that the characteristics of the signal (amplitude maxima and phase reversal) would be associated with the same cell layers in all tracks. If the pathway of volume conduction from CA1 to entorhinal cortex was across angular bundle to the deep layers of MEC one would expect the theta rhythm recorded to be greatest in amplitude in the deep layers and gradually decrease as the electrode moved toward the pial surface. This was never the case; in fact the theta recorded in angular bundle and layers VII-V of MEC was intermittent and of lower amplitude than theta rhythm recorded in layer III. If the deep MEC theta rhythm were volume conducted from CA1 along a pathway through the cellular layers due to anisotropy one would expect it to be greatest in amplitude at the medial boundary of entorhinal cortex (that portion of entorhinal cortex which is closest to CA1) and least at the lateral boundary. No systematic attenuation in amplitude of deep entorhinal theta rhythm is recorded in sequentially more lateral penetrations across medial entorhinal cortex (Fig. 6). Similar arguments can be made for volume conduction of the DG theta rhythm to superficial entorhinal cortex. Amplitude should be maximum in the deepest layers in which the signal is recorded and in the most medial portion of entorhinal cortex. As seen from the laminar profiles (Figs. 5 and 7) and the composite drawing (Fig. 6), this is not the case.

The amplitude of volume-conducted signals attentuates rapidly. Medial entorhinal cortex is at least $1.5 \mathrm{~mm}$ from CAl and dentate gyrus. If the conductivities were 
such that CAl theta rhythm could be recorded in entorhinal cortex, one would expect to record it also in isocortical areas immediately adjacent to the lateral boundary of medial entorhinal cortex. No theta rhythm is recorded there. In one animal a large isocortical lesion caused gross distortion of the entire posterior portion of the cerebral hemisphere. The lesion spared the entire hippocampal region but increased the distance between CAl (and dentate gyrus) and medial entorhinal cortex to twice the normal value. The MEC laminar profile in this animal resembled all the others; maximum amplitude theta rhythms were $230 \mu \mathrm{V}$ (deep) and $167 \mu \mathrm{V}$ (superficial).

Finally, volume-conducted theta rhythms recorded in neocortex above CA1 and in thalamus below dentate gyrus are found to be isomorphic to CAI and dentate gyrus theta rhythms respectively although the amplitude of the signal decreases with increasing distance from the generator ${ }^{50}$. Theta rhythms recorded in entorhinal cortex are not isomorphic to those recorded in CAl and dentate gyrus. Thus there is no evidence to suggest that the theta rhythm recorded in medial entorhinal cortex is volume conducted from CAl or dentate gyrus. It is not known whether or not a theta rhythm is generated in any subicular areas. Many of the arguments given above would also apply to the possibility that the medial entorhinal cortex theta rhythm was volume conducted from a generator in subicular areas.

\section{Hypotheses about MEC theta rhythm generation}

The laminar profiles show that there is at least one source and one sink of current flow associated with MEC theta rhythm. When there exist a source and a sink, the source can be the active site (presumably IPSPs) and the sink the passive site of returning current; or the sink can be the active site (presumably EPSPs) and the source passive; or both can be active (active synapses at both sites). Given these possibilities, there are many hypotheses about the generation of MEC theta rhythm. Two of these seem to us to be more attractive than the others.

The first hypothesis is that the only neuronal elements generating the transmembrane current flow comprising the sources and sinks are the pyramidal cells of layer III and their dendritic processes in layers $11 \mathrm{I}$ and I. In this case, there is one theta rhythm generator in MEC. The presence in layer III of cells which fire in rhythmic bursts correlated with the theta rhythm suggests that the active synapses of the generator may be in layer III.

The second hypothesis is that both layer III pyramidal cells and layer II star cells generate transmembrane current flow comprising the sources and sinks. In this case, there are two generators of MEC theta rhythm. The appeal of this second hypothesis arises from the intimate anatomical connections between MEC and hippocampus. The MEC layer III cells are pyramidal cells; they project to CAl pyramidal cells. The theta rhythm of MEC layer III is the same phase as CAl theta rhythm and is more similar to it in amplitude modulation and wave shape than it is to dentate gyrus theta rhythm. The MEC layer II star cells project to dentate gyrus granule cells. The theta rhythm of MEC layers II-I is the same phase as dentate gyrus theta rhythm and is more similar to it in amplitude modulation than it is to CAl theta rhythm. Afferent projections to medial entorhinal cortex suggest that cells of each layer can have unique connections 
with other brain regions. Dendritic ramification patterns of layer III pyramidal cells and layer II star cells suggest that the afferent systems which arrive at layers III and II respectively can influence only the cells of that particular layer, whereas afferents which arrive at layer I may influence either or both pyramidal cells of layer III and star cells of layer II. All this suggests a pattern of separate circuits for the projection cells of layers III and II; separate circuits which can be linked by connections in layer I. Thus an anatomical circuit associated with generation of theta rhythm in CA1 might also be associated with generation of deep MEC theta rhythm and an anatomical circuit associated with generation of theta rhythm in DG might also be associated with generation of superficial MEC theta rhythm. The anatomical evidence to date and the data in this paper make this second hypothesis more attractive than the first, but they do not confirm it.

\section{A possible pacemaker}

Since the behavioral correlate of entorhinal (deep and superficial) and hippocampal (CA1 and DG) theta rhythms appear to be identical and since the frequency modulation of all four signals is identical one might suspect that another region of brain which projects to all of these areas acts as a pacemaker for them. The medial septal nucleus and nucleus of the diagonal band of Broca have been assigned just such a role with regard to hippocampal theta rhythms.

Three lines of evidence suggest that medial septal nucleus and nucleus of the diagonal band of Broca act as a pacemaker. First, cells in these areas project to Ammon's horn and the dentate gyrus ${ }^{28,45}$. These projections are at least in part cholinergic ${ }^{22,23,29}$. Second, some cells in these areas fire in bursts, each locked to the same phase of the theta wave $11,12,32,34,36,48$. The presence of such bursting cells is a necessary condition for a pacemaker but this finding alone does not prove that septum is a pacemaker. The rhythmic firing of septal cells could result from hippocampal input to them or could have a common cause with hippocampal theta rhythm. Petsche et al. ${ }^{34}$ were able to record bursting cells in the absence of theta rhythm and McLennan and Miller ${ }^{27}$ were able to record them after fimbria cuts suggesting that in fact these cells can be pacemakers. Whether these cells are inherently rhythmic or whether they depend on synaptic drive for their rhythmicity is an unresolved question ${ }^{26,48}$. Third, and most important, lesions of the fimbria fornix or medial septal nucleus and nucleus of the diagonal band result in the disappearance of theta rhythm in the hippocampus ${ }^{13,33}$.

If these areas also pace EC they must project there and disruption of the projection must cause disappearance of MEC theta rhythm. Medial septal nucleus and nucleus of the diagonal band of Broca project to all of EC diffusely across all cortical layers $28,37,39,44$. Cholinesterase activity does exist in medial entorhinal cortex of rats; layers I, II, V-VII stain moderately for AChE, layers III and IV stain lightly43. Lesions of the medial septal nucleus cause loss of AChE at the same time (6 days) in the moderately stained layers of $\mathrm{MEC}^{29}$. There are no data about MEC slow waves after septal lesion. 
Amplitude

In our work and that of others ${ }^{49}$, the average maximum of both entorhinal theta rhythms is found to be approximately one-half to two-thirds the maximum amplitude of CAl theta rhythm when recordings are made simultaneously between the CAI or entorhinal electrodes and a distance indifferent electrode in the rat during locomotion; the maximum amplitude of dentate gyrus theta rhythm under these same conditions is one and one-half to two times that of CAl. These findings may be associated with the cell densities of the particular areas involved.

These data suggest a new perspective on the way these septal, hippocampal, and entorhinal structures function. Theta rhythm can no longer be viewed as a phenomenon uniquely associated with hippocampus, it also is associated with a major extrinsic projection to hippocampus. The septal cells which pace hippocampal theta rhythm may also pace entorhinal theta rhythm thus influencing mechanisms in both structures which gate information flow into and between them.

\section{ACKNOWLEDGEMENTS}

This research was supported by NIH Grants NS 12664; NS 14497; and NSF grant BNS 77-09375 to J. B. Ranck, Jr. and NIH Grant NS 05773 to V. E. Amassian. We wish to thank Maria Zeballos, Bobby Marsh and Larry Eberle for technical assistance. We are most grateful to Drs. Jonathan Winson, Kenneth Casey and Peter Coyle for discussions about the work and to Dr. Winson and Dr. Alan Rudell for the use of their computer programs.

\section{REFERENCES}

1 Adey, W. R., Organization of the rhinencephalon. In H. H. Jasper et al. (Eds.), Reticular Formation of the Brain, Little, Brown and Company, Boston, 1958, pp. 621-644.

2 Adey, W. R., Dunlop, C. W. and Hendrix, C. E., Hippocampal slow waves, Arch. Neurol. (Chic.), 3 (1960) 75-90.

3 Blackstad, T. W., Commissural connections of the hippocampal region in the rat, with special reference to their mode of termination, J. comp. Neurol., 105 (1956) 417-534.

4 Bland, B. H., Andersen, P. and Ganes, T., Two generators of hippocampal theta activity in rabbits, Brain Research, 94 (1975) 199-218.

5 Bland, B. H. and Whishaw, I. Q., Generators and topography of hippocampal theta (RSA) in the anaesthetized and freely moving rat, Brain Research, 118 (1976) 259-280.

6 Cajal, S. Ramón y, Studies on the Cerebral Cortex (Limbic Structures) (Trans. by L. M. Kraft), Lloyd-Luke Ltd., London, and The Yearbook Publishers Inc., Chicago, 1955.

7 Coleman, J. R. and Lindsley, D. B., Hippocampal electrical correlates of free behavior induced by stimulation of two hypothalamic-hippocampal systems in the cat, Exp. Neurol., 49 (1975) 506-528.

8 Fif ková, E., Two types of terminal degeneration in the molecular layer of the dentate fascia following lesions of the entorhinal cortex, Brain Research, 96 (1975) 169-175.

9 Geneser-Jensen, F. A. and Blackstad, T. W., Distribution of acetyl cholinesterase in the hippocampal region of the guinea pig. I. Entorhinal area, parasubiculum, and presubiculum, Z. Zellforsch., 114 (1971) $460-481$.

10 Geneser-Jensen, F. A., Haug, F.-M. S. and Danscher, G., Distribution of heavy metals in the hippocampal region of the guinea pig, $Z$. Zellforsch., 147 (1974) 441-478.

11 Gogolák, G., Petsche, H., Sterc, J. and Stumpf, C., Septum cell activity in the rabbit under reticular stimulation, Brain Research, 5 (1967) 508-510.

12 Gogolák, G., Stumpf, C., Petsche, H. and Sterc, J., The firing pattern of septal neurons and the form of the hippocampal theta wave, Brain Research, 7 (1968) 201-207. 
13 Green, J. D. and Arduini, A. A., Hippocampal electrical activity in arousal, J. Neurophysiol., 17 (1954) 533-557.

14 Green, J. D., Maxwell, D. S., Schindler, W. J. and Stumpf, C., Rabbit EEG 'theta' rhythm: its anatomical source and relation to activity in single neurons, J. Neurophysiol., 23 (1960) 403-420.

15 Green, J. D. and Petsche, H., Hippocampal electrical activity. II. Virtual generators, Electroenceph. clin. Neurophysiol., 13 (1961) 847-853.

16 Harper, R. M. and McGinty, D. J., A technique for recording single neurons from unrestrained animals. In M. I. Phillips (Ed.), Brain Unit Activity During Behavior, Charles C. Thomas, Springfield, Ill., 1973, pp. 130-154.

17 Haug, F.-M. S., Sulphide silver pattern and cytoarchitectonics of parahippocampal areas in the rat. Special reference to the subdivision of area entorhinalis (area 28) and its demarcation from the pyriform cortex, Advanc. Anat. Embryol. Cell Biol., 52 (1976) 3-73.

18 Hjorth-Simonsen, A., Projection of the lateral part of the entorhinal area to the hippocampus and fascia dentata, J. comp. Neurol., 146 (1972) 219-232.

19 Hjorth-Simonsen, A. and Jeune, B., Origin and termination of the hippocampal perforant path in the rat studied by silver impregnation, J. comp. Neurol., 144 (1972) 215-232.

20 Holmes, J. E. and Adey, W. R., Electrical activity of the entorhinal cortex during conditioned behavior, Amer. J. Physiol., 199 (1960) 741-744.

21 Krieg, W. J. S., Connections of the cerebral cortex. I. The albino rat. B. Structure of the cortical areas, J. comp. Neurol., 84 (1946) 277-323.

22 Lewis, P. R. and Shute, C. C. D., The cholinergic limbic system: Projections to the hippocampal formation, medial cortex, nuclei of the ascending cholinergic reticular system, and the subfornical organ and supra-optic crest, Brain, 90 (1967) 521-540.

23 Lewis, P. R., Shute, C. C. D. and Silver, A., Confirmation from choline acetylase analyses of a massive cholinergic innervation to the rat hippocampus, J. Physiol. (Lond.), 191 (1967) 215-224.

24 Lorente de Nó, R., Studies on the structure of the cerebral cortex. I. The area entorhinalis, $J$. Psychol. Neurol (Lpz.), 45 (1933) 381-438.

25 McFarland, W. L., Teitelbaum, H. and Hedges, E. K., Relationship between hippocampal theta activity and running speed in the rat, J. comp. physiol. Psychol., 88 (1975) 324-328.

26 McLennan, H. and Miller, J. J., The hippocampal control of neuronal discharges in the septum of the rat, J. Physiol. (Lond.), 237 (1974) 607-624.

27 McLennan, H. and Miller, J. J., Frequency-related inhibitory mechanisms controlling rhythmical activity in the septal area, J. Physiol. (Lond.), 254 (1976) 827-841.

28 Meibach, R. C. and Siegel, A., Efferent connections of the septal area in the rat : an analysis utilizing retrograde and anterograde transport methods, Brain Research, 119 (1977) 1-20.

29 Mellgren, S. I. and Srebro, B., Changes in acetylcholinesterase and distribution of degenerating fibres in the hippocampal region after septal lesions in the rat, Brain Research, 52 (1973) 19-36.

30 Mitchell, S. J. and Ranck, J. B., Jr., Firing patterns and behavioral correlates of neurons in entorhinal cortex of freely-moving rats, Neurosci. Abstr., 3 (1977) 202.

31 Nafstad, P. H. J., An electron microscope study on the termination of the perforant path fibers in the hippocampus and the fascia dentata, Z. Zellforsch., 76 (1967) 532-542.

32 Petsche, H., Gogolák, G. and van Zwieten, P. A., Rhythmicity of septal cell discharges at various levels of reticular excitation, Electroenceph. clin. Neurophysiol., 19 (1965) 25-33.

33 Petsche, H. and Stumpf, C., Topographic and toposcopic study of origin and spread of the regular synchronized arousal pattern in the rabbit, Electroenceph. clin. Neurophysiol., 13 (1960) 589-600.

34 Petsche, H., Stumpf, C. and Gogolák, G., The significance of the rabbit's septum as a relay station between the midbrain and the hippocampus, I. The control of hippocampal arousal activity by the septum cells, Electroenceph. clin. Neurophysiol., 14 (1962) 202-211.

35 Ranck, J. B., Jr., Studies on single neurons in dorsal hippocampal formation and septum in unrestrained rats. Part 1. Behavioral correlates and firing repertoires, Exp. Neurol., 41 (1973) 461-531.

36 Ranck, J. B., Jr., Behavioral correlates and firing repertoires of neurons in septal nuclei in unrestrained rats. In J. F. DeFrance (Ed.), The Septal Nuclei, Plenum Press, New York, 1976, pp. 423462.

37 Segal, M., Afferents to the entorhinal cortex of the rat studied by the method of retrograde transport of horseradish peroxidase, Exp. Neurol., 57 (1977) 750-765.

38 Shipley, M. T., Geneser-Jensen, F. A. and Meier, A., Correlated histochemical and experimental evidence for a subdivision of the entorhinal area of the guinea pig, Cell Tiss. Res., 150 (1974) 455462. 
39 Siegel, A. and Tassoni, J. P., Differential efferent projections of the lateral and medial septal nuclei to the hippocampus in the cat, Brain Behav. Evol., 4 (1971) 201-219.

40 Steward, O., Topographic organization of the projections from the entorhinal area to the hippocampal formation of the rat, J. comp. Neurol., 167 (1976) 285-314.

41 Steward, O. and Scoville, S. A., Cells of origin of entorhinal cortical afferents to the hippocampus and fascia dentata of the rat, J. comp. Neurol., 169 (1976) 347-370.

42 Steward, O., Scoville, S. A. and Vinsant, S. L., Analysis of collateral projections with a double retrograde labeling technique, Neurosci. Lett., 5 (1977) 1-5.

43 Storm Mathisen, J. and Blackstad, T. W., Cholinesterase in the hippocampal region, Acta anat. (Basel), 56 (1964) 216-153.

44 Swanson, L. W. and Cowan, W. M., An autoradiographic study of the organization of the efferent connections of the hippocampal formation in the rat, J. comp. Neurol., 172 (1977) 49-84.

45 Vanderwolf, C. H., Hippocampal electrical activity and voluntary movement in the rat, Electroenceph. clin. Neurophysiol., 26 (1969) 407-418.

46 Van Hoesen, G. W. and Pandya, D. N., Some connections of entorhinal (area 28) and perirhinal (area 35) cortices of the rhesus monkey. I. Temporal lobe afferents, Brain Research, 95 (1975) 1-24.

47 Whishaw, I. Q. and Vanderwolf, C. H., Hippocampal EEG and behavior: Changes in amplitude and frequency of RSA (theta rhythm) associated with spontaneous and learned movement patterns in rats and cats, Behav. Biol., 8 (1973) $461-484$.

48 Wilson, C. L., Motter, B. C. and Lindsley, D. B., Influences of hypothalamic stimulation upon septal and hippocampal electrical activity in the cat, Brain Research, 197 (1976) 55-68.

49 Winson, J., Patterns of hippocampal theta rhythm in the freely-moving rat, Electroenceph. clin. Neurophysiol., 36 (1974) 291-301.

50 Winson, J., Hippocampal theta rbythm. I. Depth profiles in the curarized rat, Brain Research, 103 (1976) $57-70$.

51 Winson, J., Hippocampal theta rhythm. II. Depth profiles in the freely moving rabbit, Brain Research, 103 (1976) 71-79. 